\title{
Regional anesthesia: sympathectomy-mediated vasodilation
}

\author{
Steven B. Backman, MDCM, PhD
}

Received: 23 April 2009/Accepted: 11 June 2009/Published online: 28 July 2009

(c) Canadian Anesthesiologists' Society 2009

\section{To the Editor,}

I read with interest the recent study concerning autonomic, sensory, and motor effects of lumbar epidural anesthesia in patients randomized to receive varying bupivacaine doses and concentrations. ${ }^{1}$ At lower concentrations, sensory block was observed in the mid-thoracic dermatomes with little change in the temperature or pulse oximeter perfusion index of the upper and lower extremities despite a concomitant decrease in systemic arterial pressure. These data were interpreted to suggest that sensory neural function may, in some cases, "be more susceptible than sympathetic neural function to local anesthetic blockade", in contrast to widely accepted dogma that the reverse is the case.

As alluded to in the Discussion, these data must be interpreted in the context of direct (sympatholysis secondary to local anesthetic effects on spinal sympathetic outflow) and indirect (e.g., baroreceptor-mediated sympathetic activation secondary to fall in systemic arterial pressure) effects on autonomic tone. Thus, the response of sympatho-target organs, as described in this report, will reflect the balance of these opposing effects. A further confounding variable rarely addressed in the literature is that a dermatome may not reflect the segmental organization of sympathetic outflow to that area. A simple example to consider is the thumb, which has sensory innervation from spinal segments $\mathrm{C} 6$, but sympathetic preganglionic neurons are not found rostral to T1. In fact, there is extensive convergence and divergence of input from sympathetic preganlionic axons onto postganglionic cells contained in the sympathetic chain, and sympathetic target organs may

S. B. Backman, MDCM, PhD ( $)$

McGill University, Montreal, Canada receive input from numerous spinal cord segments. ${ }^{2}$ This may be one mechanism to facilitate a "widespread diffusion of nervous impulses" associated with the sympathetic response to stress. Of note, vasoconstriction of the upper extremity is mediated by sympathetic preganglionic neurons from the middle to the caudal thoracic spinal segment, the axons of which course in the sympathetic chain before synapsing onto sympathetic (vasoconstrictor) postganlionic neurons in the stellate ganglion. ${ }^{3}$ Thus, neuraxial anesthesia producing a sensory deficit in dermatome $\mathrm{T} 8$ may indeed be accompanied by vascular resistance changes in the upper extremity simply on the basis of anatomy: both afferent activity and autonomic outflow are blocked at the same segmental level. To conclude that this is a result of extraordinary sensitivity of sympathetic outflow to local anesthetic action, as has been decided previously, ignores these anatomical features that have been known for more than a century. ${ }^{3}$

A further consideration involves the unique physiological properties of sympathetic preganglionic neurons, such as tonic activity, refractory period, and axonal fibre diameter, which distinguish them from spinal sensory and motor neurons. ${ }^{4}$ These properties may account, in part, for the differential blocking effects of local anesthetics on specific types of spinal neurons. Moreover, functionally specific subgroups of sympathetic neurons may themselves be differentiated on the basis of their physiological properties that are particularly matched to the behaviour (e.g. frequency-response characteristics) of their sympathotarget organs. ${ }^{5}$

The above points may be relevant when contemplating the differential effects of neuraxial (spinal or epidural) anesthesia on autonomic, sensory, and motor responses.

Competing interests None declared. 


\section{References}

1. Ginosar $Y$, Weiniger $C F$, Kurz $V$, Babchenko A, Nitzan $M$, Davidson E. Sympathectomy-mediated vasodilatation: a randomized concentration ranging study of epidural bupivacaine. Can J Anesth 2009; 56: 213-21.

2. Skok VI. Physiology of Autonomic Ganglia. Tokyo: Igaku Shoin Ltd; 1973.

3. Backman SB, Stein RD, Polosa C. Organization of the sympathetic innervation of the forelimb resistance vessels in the cat. Anesth Analg 1999; 88: 320-5.

4. Backman SB, Henry JL. Physiological properties of sympathetic preganglionic neurons in the thoracic intermediolateral nucleus of the cat. Can J Physiol Pharmacol 1984; 62: 1183-93.

5. Janig W, Habler HJ. Neurophysiological analysis of target-related sympathetic pathways - from animal to human: similarities and differences. Acta Physiol Scand 2003; 177: 255-74.

\section{Reply}

We thank Dr. Backman for his interest in our manuscript and for his erudite description of differential sympathetic and sensory neural innervation of the upper limb and its effect on the observed response to local anesthetic blockade. As he correctly identifies, these principles have been understood for many years. However, we stated quite clearly in the discussion section of our article that "this was not an electrophysiological study, and we do not make inferences regarding the susceptibilities of different sympathetic, sensory, and motor nerves to local anesthetic blockade. We have deliberately confined discussion to the effect on sympathetic, sensory, and motor function following epidural anesthesia."

Neither was ours an anatomical study. We designed a clinical study (a concentration-ranging study of lumbar epidural bupivacaine) to assess a daily clinical challenge, i.e., monitoring for signs of sympathetic, sensory, and motor blockade following regional anesthesia. ${ }^{1}$ We used both cutaneous temperature and quantitative photoplethysmography (pulse oximeter-derived perfusion index) as clinical tools that may be available in a standard operating room. We have demonstrated elsewhere that the pulse oximeter-derived perfusion index is the earliest and most sensitive of bedside indices to detect sympathectomymediated vasodilatation in the foot. ${ }^{2}$
We used both vasodilatation in the toe and compensatory vasoconstriction in the finger as indices of the extent and intensity of sympathetic block, as compensatory vasoconstriction in the hand has been described by others. ${ }^{3,4}$ These authors reasoned that upper limb vasoconstriction reflects the effect of generalized sympathetic discharge on unblocked cervical or high thoracic sympathetic afferents and presumably contributes to the conservation of core temperature and arterial pressure. The insights from Dr. Backman's letter further refine this understanding.

Within the stated limitations of our study, we observed that the extent of sympathectomy-mediated vasodilatation in the foot and vasoconstriction in the hand was determined more by drug concentration than by drug dose, and we noted that sensory neural function was blocked more noticeably than sympathetic neural function at dilute epidural bupivacaine concentrations. We believe that these observations are of clinical relevance, particularly as they provide further support for the current use of dilute local anesthetic mixtures in epidural techniques for labour and postoperative analgesia.

Competing interests None declared.

\section{References}

1. Ginosar $Y$, Weiniger $C F$, Kurz $V$, Babchenko A, Nitzan $M$, Davidson E. Sympathectomy-mediated vasodilatation: a randomized concentration ranging study of epidural bupivacaine. Can J Anesth 2009; 56: 213-21.

2. Ginosar $Y$, Weiniger CF, Meroz Y, et al. Pulse oximeter perfusion index as an early indicator of sympathectomy after epidural anesthesia. Acta Anaesthesiol Scand 2009; Epub ahead of print. doi:10.1111/j.1399-6576.2009.01968.x.

3. Bengtsson M. Changes in skin blood flow and temperature during spinal analgesia evaluated by laser Doppler flowmetry and infrared thermography. Acta Anesthesiol Scand 1984; 28: 625-30.

4. Matsukawa T, Sessler DI, Christensen R, Ozaki M, Schroeder M. Heat flow and distribution during epidural anesthesia. Anesthesiology 1995; 83: 961-7.

Yehuda Ginosar, MD

Elyad Davidson, MD

Hadassah Hebrew University Medical Center, Jerusalem, Israel 